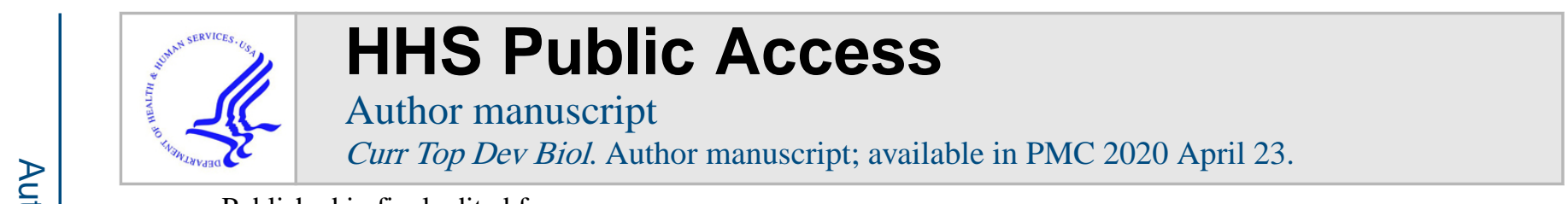

Published in final edited form as:

Curr Top Dev Biol. 2020 ; 136: 219-242. doi:10.1016/bs.ctdb.2019.09.001.

\title{
Tunicate Gastrulation
}

Konner M. Winkley ${ }^{1}$, Matthew J. Kourakis² ${ }^{2}$ Anthony W. DeTomaso², Michael T. Veeman ${ }^{1}$, William C. Smith²

1.Division of Biology, Kansas State University, Manhattan, KS 66506

2.Department of Molecular, Cellular and Developmental Biology, University of California, Santa Barbara, CA 93106

\begin{abstract}
Tunicates are a diverse group of invertebrate marine chordates that includes the larvaceans, thaliaceans, and ascidians. Because of their unique evolutionary position as the sister group of the vertebrates, tunicates are invaluable as a comparative model and hold the promise of revealing both conserved and derived features of chordate gastrulation. Descriptive studies in a broad range of tunicates have revealed several important unifying traits that make them unique among the chordates, including invariant cell lineages through gastrula stages and an overall morphological simplicity. Gastrulation has only been studied in detail in ascidians such as Ciona and Phallusia, where it involves a simple cup-shaped gastrula driven primarily by endoderm invagination. This appears to differ significantly from vertebrate models, such as Xenopus, in which mesoderm convergent extension and epidermal epiboly are major contributors to involution. These differences may reflect the cellular simplicity of the ascidian embryo.
\end{abstract}

\section{Introduction: Tunicates: their place on the evolutionary tree and their contribution to our understanding of embryology}

Tunicates were among the earliest experimental models for embryology. Embryologists were attracted to the ascidian embryo, with its regular cleavage program and small, simple embryonic body plan. Laurent Chabry performed blastomere separation experiments in early embryos of the ascidian Ascidiella aspersa that are regarded as foundational to the discipline of experimental embryology (Chabry, 1887; Fischer, 1992). He found that isolated blastomeres showed predetermined fates, dividing as if they were still in the intact embryo. Although not obvious in their diverse adult forms, tunicates embryos are unmistakably chordate with a notochord and dorsal hollow nerve cord. The close evolutionary relationship of ascidians to vertebrates was well appreciated by this time (Darwin, 1871; Haeckel, 1874; Kowalevsky, 1866). Edwin G. Conklin (Conklin, 1905a) built on Chabry's work and mapped the complete lineage of cells through and beyond gastrulation, with illustrations by embryonic stage and a nomenclature still in use. Conklin's work included descriptions of cleavage planes, cell-cell contacts, nuclear positions, distribution of cytoplasmic determinants, cell fates, polar body location, and spindle dynamics, as well as comparisons of gastrulation and other aspects of embryogenesis between ascidians and other animals. Noriyuki Satoh's SEM studies of Halocynthia roretzi confirmed and expanded on these early descriptions of ascidian development, bolstering inferences concerning the coordination of 
cell movements during gastrulation and neurulation and revealing additional surface features, such as the presence of filopodia from interior membranes over the blastocoel (Satoh, 1978).

Since that time, molecular approaches in Halocynthia, Ciona and Phallusia have revealed much about the mechanisms driving ascidian patterning and morphogenesis, but little is known about tunicate gastrulation and other developmental processes outside these ascidian models. The tunicates are thought to have diverged from their last common ancestor with the vertebrates more than 500 million years ago, and are a large and diverse group. They are usually regarded as a chordate sub-phylum, but some authors have argued that they should be elevated to phylum status (Satoh et al., 2014). The ascidians (class Ascidiacea) are sessile as adults and are the most speciose group of tunicates, represented by nearly 3000 separate types ("WoRMS - World Register of Marine Species," 2019) across three orders, Phlebobranchia, Stolidobranchia and Aplousobranchia, each characterized by differences in gill-slit morphology. Many of these are solitary, but colonialism has evolved independently in multiple ascidian lineages (Brown and Swalla, 2012), with varying degrees of physiological integration. The thaliaceans (class Thaliacea), with about 80 known species, are pelagic as adults and move through the water column by pulsating muscular contractions, using incurrent and excurrent siphons at opposite ends of the body. Thaliaceans, including pyrosomes, salps and doliolids, can be seen singly, or as chains of asexually budding animals. They can show alternation of sexual and asexual generations, and can reproduce asexually at an explosive rate during plankton blooms (Holland, 2016). The larvaceans (class Appendicularia) are also pelagic and represented by about 70 species ("WoRMS - World Register of Marine Species," 2019). Larvaceans are so called because they never lose their tails and resemble larvae into adulthood. They live inside buoyant glycoprotein "houses" that aid in filter feeding and are secreted by specialized epidermal organs.

Molecular phylogenies using concatenated sequence datasets reveal that tunicates, and not cephalochordates, are the sister taxon to the vertebrates (Figure 1) (Bourlat et al., 2009; Delsuc et al., 2006). Two recent phylogenies, largely congruent, go further to resolve relations amongst the tunicates (Delsuc et al., 2018; Kocot et al., 2018) as summarized in Figure 1. Larvaceans are the basal lineage, pointing to a possible pelagic ancestor for the tunicates as a whole. The ascidian order Stolidobranchia is sister to a group that includes the Thaliacea plus an Aplousiobranchia/Phlebobranchia pairing (the phlebobranch ascidians may themselves be paraphyletic, however). The placement of thaliaceans within the Ascidiacea is contrary to most older classification schemes of the last century (see, e.g., (Brusca and Brusca, 1990)) but is well-supported and suggests this free-swimming group evolved from a benthic ascidian ancestor, underscoring the potential for extensive morphological innovation in these lesser-studied tunicates (Kocot et al., 2018).

\section{Events leading up to gastrulation Cleavage patterns}

Only minor differences in embryonic cleavage patterns have been described between different ascidian species. Ciona cell lineages were first described in detail by Conklin 
(Conklin, 1905a), who also took advantage of the highly pigmented Styela egg to identify a cytoplasmic component he called the "myoplasm" that segregates with muscle fate (Conklin, 1905b). This was arguably the first cytoplasmic determinant of cell fate to be identified, and provided the foundation for the mosaic theory of development (Conklin, 1905c). Conklin's cell lineages were refined and extended by Hiroki Nishida who performed blastomere labeling experiments in Halocynthia, which has a larger embryo better suited to single-cell microinjection than Ciona (Nishida, 1987; Nishida and Satoh, 1985, 1983). Most cells are restricted to a single tissue fate by the start of gastrulation at the 112-cell stage (Nishida, 1987), though there is extensive subsequent refinement of cell identity in the neural lineages in particular (Hudson et al., 2007; Racioppi et al., 2014; Stolfi et al., 2011). The fate map has not been deciphered with single cell resolution all the way to the hatched larval stage, but several specific lineages have been traced well past gastrulation (Carlson et al., 2015; Nicol and Meinertzhagen, 1988a; Stolfi et al., 2015; Stolfi and Levine, 2011; Tokuoka et al., 2004; Wang et al., 2019). Many markers of tissue differentiation are expressed normally even in embryos that have been cleavage arrested prior to gastrulation (Nishikata et al., 1987; Okado and Takahashi, 1988; Whittaker, 1973).

The first two rounds of division are at right angles and give rise to four similarly sized daughter cells. The first division splits the left and right halves of the embryo, and the second division nominally separates anterior from posterior (Conklin, 1905a). The third round of division is again orthogonal to the prior two and separates the animal and vegetal hemispheres of the embryo. These cleavages are slightly asymmetric in volume, with the animal daughters all somewhat smaller than their vegetal siblings (Conklin, 1905a). Cell divisions remain synchronous through the $4^{\text {th }}$ and $5^{\text {th }}$ cell cycles, but many of the vegetal cell divisions are highly asymmetric with major differences in size and fate between anterior and posterior daughters (Conklin, 1905a; Dumollard et al., 2017; Hibino et al., 1998; Iseto and Nishida, 1999; Nishikata et al., 1999; Patalano et al., 2006; Tassy et al., 2006). After the 32cell stage, cell cycle times slow in the vegetal hemisphere to give distinct 44 and 76-cell stages (Dumollard et al., 2013). Animal cells continue to divide relatively symmetrically to give rise to most of the ectoderm, whereas many of the vegetal divisions are asymmetric in fate and/or size to give rise to a variety of largely mesodermal and endodermal cell types (Conklin, 1905a; Nishida, 1987).

\section{Ooplasmic segregation/PEM}

Early embryonic patterning depends on two successive cytoplasmic reorganizations in the first cell cycle known as the first and second ooplasmic segregations. Ascidian oocytes are polarized along the animal-vegetal axis prior to fertilization (Sardet et al., 1992), and the first ooplasmic segregation helps to reinforce this polarity. The second ooplasmic segregation breaks the radial symmetry of the egg and establishes the future anteriorposterior axis. Both phases involve the movement of a class of maternally deposited RNAs collectively known as the postplasmic/PEM (Posterior End Mark) RNAs (reviewed in (Prodon et al., 2007)) along with mitochondria rich myoplasm and cortical endoplasmic reticulum (ER). The first phase of ooplasmic segregation is influenced by both the animalvegetal polarization of the oocyte prior to fertilization as well as the site of sperm entry. Sperm entry triggers first a calcium wave and a subsequent contraction wave that 
concentrates the postplasmic/PEM RNAs and associated factors at the vegetal pole (McDougall and Sardet, 1995; Speksnijder et al., 1990).

The second phase of ooplasmic segregation is driven by sperm aster microtubules and cortical microfilaments (Chiba et al., 1999). It relocates the vegetal PEM components to a more equatorial position that defines what is widely referred to as the future posterior side of the embryo (Sardet et al., 1989). As in many species, however, this new axis of organization orthogonal to the AV axis is not perfectly congruent with any Cartesian axis of the hatched larva. The cortical ER, postplasmic/PEM RNAs, and myoplasm are inherited by the left and right posterior vegetal blastomeres at the eight-cell stage, and aggregate to form a structure called the centrosome attracting body (CAB) (Hibino et al., 1998). The CAB is continuously partitioned into the posterior-most vegetal cells during the subsequent rounds of division and is responsible for the unequal cleavages characteristic of these lineages (Patalano et al., 2006). Much of the anterior-posterior patterning of the embryo is thought to result directly or indirectly from the asymmetric inheritance of the CAB and the PEM RNAs. This includes a Zic family transcription factor variously known as Macho1 or Zic-r.a that has been identified as one of the key components of Conklin's myoplasm (Nishida and Sawada, 2001).

\section{Origin of germ layers}

As seen in diverse metazoans (Logan et al., 1999; Miyawaki et al., 2003; Onai, 2019; Wikramanayake et al., 2003), maternal nuclear $\beta$-catenin plays a key role in ascidian germ layer specification. $\beta$-catenin signaling is required for endodermal cell fate in the most vegetal cells as well as mesodermal cell fates in the more marginal vegetal cells. In Ciona and other ascidians, this involves two successive $\beta$-catenin dependent fate specification events. Nuclear $\beta$-catenin is observed in all the mesendodermal precursors at the 16-cell stage and is required for mesendodermal fate (Imai et al., 2000). In the next cell cycle, $\beta$ catenin becomes restricted to only the most vegetal daughters and is required for the segregation of endoderm from marginal fates including mesoderm and some neural ectoderm (Hudson et al., 2013). In the first specification event, nuclear localization of $\beta$ catenin in the vegetal cells is antagonistic to the ubiquitous expression of the maternal transcription factor Gata.a. Gata.a promotes the transcription of ectodermal specific genes in the animal cells (Rothbacher et al., 2007), while the nuclear localization of $\beta$-catenin both prevents the expression of ectodermal genes and promotes expression of mesendodermal genes in the vegetal cells (Imai et al., 2016). The second specification event in the following cell cycle also involves antagonism between vegetal $\beta$-catenin signaling and more broadly expressed Gata.a (Imai et al., 2016), where $\beta$-catenin promotes endodermal fate. The precise mechanisms linking preexisting oocyte AV polarity and ooplasmic segregation to $\beta$-catenin stabilization and nuclear entry remain unclear. Several downstream targets of $\beta$-catenin have been identified, including vegetally-expressed FGFs that are involved in a broad range of cell fate decisions at the margin of the embryo (Hudson et al., 2016; Imai, 2003). 


\section{Mechanisms of gastrulation}

Most ascidians initiate gastrulation after the seventh cell cycle. For Ciona this is $\sim 5$ hours after fertilization at $18^{\circ} \mathrm{C}$ (Hotta et al., 2007). The embryo rapidly flattens along the animalvegetal axis and invaginates to form a cup-shaped early gastrula (Figure 2, left). The blastopore constricts over $\sim 2$ hours to bring not just the endoderm but also the notochord, muscle and mesenchyme precursors into the interior of the embryo (Figure 2, right).

\section{Endoderm-intrinsic forces in gastrulation}

The most detailed mechanistic description of forces driving the morphogenetic events of early tunicate gastrulation used a combination of fixed and live imaging in four different ascidian species (Ciona intestinalis type A [now known as Ciona robusta], Ciona savignyi, Phallusia mammilata, and Boltenia villosa), all of which show essentially superimposable cleavage patterns and cell-shape changes during gastrulation (Sherrard et al., 2010). This study generated a comprehensive model of invagination through observation, experimental manipulation and computer simulation. One surprising finding was that force-generating myosin II dynamics intrinsic to the endoderm cells is sufficient to account for invagination. Equally surprising was the finding that the concomitant cell-shape changes such as epiboly outside the endoderm not only do not to contribute to invagination but actually oppose it. This is unlike Xenopus (Keller et al., 2003), where ectodermal epiboly is thought to help drive endodermal internalization.

Endoderm invagination is the product of two distinct and sequential steps, both driven by myosin II. In the first step the apical (vegetal-most) surfaces of the endoderm cells contract between the late 64-cell and early 112-cell stages (Apical Constriction, Figure 3). This step is characterized by the accumulation of activated dynamic myosin II complexes at the contracting apical surfaces, as seen from immunostaining for ser19-phosphorylated myosin II regulatory light chain (1P-myosin; (Sherrard et al., 2010)). This apical constriction is regulated by the well-described RhoA/Rho-kinase pathway (Beane et al., 2006; van Eekelen et al., 2010), and can be blocked by either dominant negative RhoA or pharmacological inhibition of Rho-kinase.

The second step involves embryonic morphology shifting from a flattened disk to a cup-like shape (Collared Rounding, Figure 3). This involves the endoderm cells contracting in the apical-basal dimension to become shorter, while at the same time expanding their basal surfaces contacting the overlying ectoderm. This change in contractility is observed as a redistribution of 1P-myosin staining to the basal and lateral surfaces. Interestingly, this second step is not RhoA dependent. The mechanism that regulates the transition from step 1 to step 2 and that directs the assembly of activated myosin II in the apico-basal axis remains to be determined, but a recent preprint suggests that Nodal and Ephrin signaling may be important (Fiuza et al., 2018).

Importantly, during Step 2 the apical surfaces remain constricted, although no further constriction is observed. Immunostaining shows that at the apical surfaces the more dynamic 1P-myosin is replaced with the more stable 2P-myosin which is phosphorylated on both ser19 and thr18 of the regulatory light chain. Pharmacological inhibition of RhoA at later 
steps of gastrulation causes the apical constrictions to relax, indicating a persistent requirement for this pathway. Collared rounding can occur even if the cell division from 64 to 112 cells is inhibited, indicating that this mechanism is robust.

Endoderm invagination in ascidian gastrulation involves changes in cell shape but no changes in cell-cell contacts. This is unlike many metazoans, where involution, ingression, intercalation, migration and other large scale tissue rearrangements lead to major changes in cell-cell neighbor relationships. Even in sea urchins, which have a relatively simple gastrulation, the mesenchyme cells delaminate and migrate, and the invaginating endoderm cells also undergo radial intercalation (Ettensohn, 1999, 1985). We speculate that the morphological simplicity of tunicate gastrulation reflects both the small size of the embryo and its very rapid development.

The Sherrard study dived deep into the molecular and biophysical mechanisms of early ascidian gastrulation as the embryo changes from ball-shaped to cup-shaped. Later aspects of gastrulation have not been studied in any detail. It is unknown whether notochord, muscle and mesenchyme are participating more actively later in gastrulation, and the mechanisms driving blastopore closure have not been investigated.

\section{Gastrulation in other tunicates}

As detailed in the Introduction, tunicates are a diverse group of animals with dramatically different larval and adult morphologies. Given this diversity, it is reasonable to assume that the cellular and molecular mechanisms of gastrulation may also vary. Embryos of the ascidian Halocynthia roretzi, for example, are much larger than Ciona embryos, and have minor differences in early cleavage patterns. Halocynthia gastrulation begins at the 110-cell stage (H Nishida, 1986) instead of the 112-cell stage as seen in Ciona. By contrast, the larvacean Oikopleura dioica, begins gastrulation at the 32-cell stage less than two hours after fertilization. Oikopleura has exceptionally rapid development, with the tadpole larvae hatching in as little as three hours following fertilization (Fujii et al., 2008; Nishida, 2008). Gastrulation in $O$. dioica progresses in two phases. In the first phase, the vegetal-most eight cells invaginate, with the lateral four cells proceeding the central four endoderm cells. In the second phase, cells lateral and posterior cells invaginate. Interestingly, no change to the overall spherical shape of the embryos is evident during gastrulation. Moreover, because of the small number of invaginating cells in the gastrula, there is no obvious blastopore or archenteron. Cellular mechanisms driving invagination in $O$. dioica are not known, but it has been speculated that myosin II-driven apical constriction is important (Fujii et al., 2008).

\section{Colonial tunicates}

A subset of ascidian species are colonial and grow by repeated rounds of asexual reproduction (budding) that eventually give rise to a colony of genetically identical individuals, called zooids, arranged within a common tunic. Each zooid can be sexually mature, but colonial reproduction has several notable differences when compared to solitary species, and these have hampered embryological studies. First, all colonial ascidians are brooders, and fertilization and development occur inside the adult zooids, often with maternal contributions, such as a brood pouch, which can be difficult to replicate in vitro. 
Development is also significantly slower, on the order of days to a week or more. Finally, the eggs and developing embryos are opaque, hindering high-resolution studies of cell movement. Thus little is known about gastrulation in these species, and there are no recently published studies.

More germane to this review is the potential role of a gastrulation-like process during asexual development. During the budding process, colonial ascidians are regenerating all tissues and organs from a population of pluripotent stem cells. An important question is whether asexual development is controlled by novel genes and gene regulatory networks, or if embryonic pathways and networks are redeployed (Tiozzo et al., 2008). Interestingly, there are many colonial species scattered throughout the class Ascidiacea, including different monophyletic orders, suggesting that coloniality has arisen independently multiple times. Comparison of these species reveal a diversity of budding modes, including the source of the new body, and the timing of various developmental landmarks (Tiozzo et al., 2008). However, in all species studied to date, asexually developing zooids go through an initial blastula-like stage. This is followed by invaginations and evaginations of the epithelium to form various tissues and organs. While in a sense this resembles gastrulation, these movements do not result in segregation of cells into presumptive germ layers, as they do in embryogenesis. The only species in which asexual budding has been studied at a molecular level is Botryllus schlosseri (Manni et al., 2019). In an elegant study by Ricci, et al. (Ricci et al., 2016), it was found that there is regionalization of germ-layer specific transcription factors in the blastula-like stage and during these epithelial folding events, but they do not correspond to the regions that are folding (i.e., it does not appear that the movement is correlated to germ layer specification). In this study it was found that germlayer restricted transcription factors, such as Otx (ectoderm), Fox-A1 (endoderm) and Gsc (mesoderm) are expressed in distinct regions of the blastula-like structure and could be followed through organogenesis. Importantly, the expression of each of these germ layer markers corresponded to the source of mature organs during embryogenesis. For example, the endoderm marker Fox-A1 was initially expressed in a region of the blastula-like vesicle that eventually became the gut, and results were equivalent for ectodermal and mesodermal markers.

Given that coloniality has arisen multiple times, it would hardly be feasible that completely novel mechanisms for regeneration of every tissue and organ also evolved multiple times, and a more parsimonious explanation is that this is due to the ability to coopt embryonic pathways at different times during regeneration. Interestingly though, this does not appear to include a clear recapitulation of embryonic gastrulation.

\section{The thaliaceans: salps, doliods and pyrosomes}

The thaliaceans include three orders of pelagic tunicates: the Pyrosomida, Salpida, and Doliolida. The thaliaceans are more closely related to the ascidians than to the appendicularians (see Introduction), and a conserved anatomy is evident between these two groups, particularly the large pharyngeal basket used for filter feeding in both adult ascidians and thaliaceans. Although the anatomy and reproductive cycles of thaliaceans have been described in reports dating back to the nineteenth century (see review (Piette and Lemaire, 
2015)), much remains unknown about this group of animals. Thaliaceans are more difficult to collect and culture than ascidians, and they do not readily lend themselves to modern experimental methods. Despite their similarities to ascidians, the thaliaceans have diverged extensively and have evolved complex reproductive strategies involving alternating sexual and asexual generations (Holland, 2016; Piette and Lemaire, 2015). Like some ascidian species, salps and pyrosomes can form large colonies comprised of individual zooids produced by asexual budding. While asexual budding and coloniality appear to have evolved independently several times in the ascidians (Brown and Swalla, 2012), the evolutionary history of thaliacean budding is less clear and it is uncertain whether it arose before or after the split with ascidians (see discussion in (Piette and Lemaire, 2015).

Embryonic development of salps appears to be particularly unusual. The salp lifecycle starts with the sexually produced embryo that goes on to form a single 'oozooid.' The adult oozoid then buds asexually to produce connected chains of 'blastozooids' known as 'aggregates.' Each blastozooid has germ cells that produce first eggs and later sperm. Depending on the species, only a single egg may be produced per blastozooid. Fertilized eggs attach to the adult body wall and develop viviparously. The salp zygote is called the 'blastophore' and its development remains poorly described, particularly with modern molecular tools. A 1960sera description of blastophore development in Salpa fusiformis shows a developing embryo largely unrecognizable to those familiar with ascidian or larvacean embryos (Sutton, 1960); Figure 4). It lacks an obvious notochord or neural tube, and does not produce a tadpole larva. Remarkably, it does not obviously gastrulate. Sutton identified a mass of blastophore cells that he terms 'neurochordal,' but these fail to form an identifiable notochord or neural plate and molecular markers of cell identity have not been examined in these cells. The only morphogenic event that might be conflated with gastrulation is the formation of the neurochordal invagination (N-C.I. in Figure 4). Sutton described the neuro-chordal invagination as the result of infolding caused by the differential growth of adjacent epithelia, but it is unclear whether it bears any relationship to gastrulation or neurulation. Finally, although description of embryonic development of doliolids and pyrosomes is still incomplete, neither are viviparous, and both reproduce sexually as well as asexually by budding (Holland, 2016; Lemaire, 2011; Lemaire and Piette, 2015). Although neither is thought to show the extreme derivation of development observed in salps, various species within both groups show high, but variable, degrees of degeneration of the tadpole larva morphology. For example, Doliolum larvae are reported to make a rudimentary notochord flanked by tail muscle, but no obvious nervous system (Holland, 2016). How these altered larval morphologies may be reflected in earlier gastrula morphologies is currently unknown.

\section{Peri-gastrulation events}

\section{Notochord development}

As in vertebrate embryos, early ascidian notochord morphogenesis overlaps with gastrulation. The notochord is divided into two separate developmental lineages. The primary notochord is derived from anterior vegetal (A-line) blastomeres while the secondary notochord is derived from posterior vegetal (B-line) blastomeres \{Nishida, 1987 \#18\}. Primary notochord fate is induced at the 64-cell stage and secondary notochord at the 112- 
cell stage via distinct but overlapping mechanisms (Hudson and Yasuo, 2006; Picco et al., 2007; Yasuo and Hudson, 2007). At the onset of gastrulation, the four primary notochord cells have divided once to form an arc of eight cells that are not yet contiguous with the two secondary notochord precursors (Figure 2, yellow asterisks). The two final notochord cell divisions take place during gastrulation. The primary notochord cells are effectively on the anterior lip of the blastopore during the second-last division, and invert during gastrulation so that their previously vegetal surfaces become animal. The final primary notochord division takes place while the blastopore is closing, and is immediately followed by the onset of mediolateral intercalation behaviors that cause the notochord to converge and extend into a single-file column (Munro and Odell, 2002a, 2002b). This is in striking contrast to organisms such as Drosophila where the cell cycle pauses in cells that are actively gastrulating (Wieschaus, 2000).

Perhaps the most surprising conclusion from (Sherrard et al., 2010) is the apparent absence of a contribution from the axial mesoderm (notochord and tail muscle precursors) to the forces that drive invagination during ascidian gastrulation - at least up through the formation of the cup-shaped gastrula. Although it is possible that the developing axial mesoderm could play a role in later steps, such as blastopore closure, observations of mutant Ciona, as well as tailless variants of the Molgulid species of ascidians, suggest otherwise. As detailed above, ascidians notochord morphogenesis initiates at gastrulation, although extensive convergent extension and elongation of the ascidian notochord is observed most prominently in the neurula embryo (i.e., following the completion of gastrulation) (Smith, 2018). Consistent with the absence of a role for axial mesoderm in invagination, Ciona embryos homozygous for the mutation chobi have a severe defect in notochord specification due to a mutation in the gene brachyury (Chiba et al., 2009), but nevertheless successfully complete gastrulation and properly close the blastopore. In fact, presumptive notochord cells in chobi are transfated to endoderm, further evidencing the robustness of the endoderm invagination mechanisms to perturbations (Sherrard et al., 2010), in this case to increased cell number. Likewise, a number of species of ascidians have been described that differ from the conventional body plan in having no tail, and reduced numbers of notochord and tail muscle cells- the best described of these being Molgula occulta (Swalla et al., 1993; Swalla and Jeffery, 1990). Nevertheless, these tailless species gastrulate and close their blastopores. In M. occulta, as well as the congeneric tailless species Molgula tectiformi, the notochord lineage is present in the blastula embryo, as apparent by the expression of brachyury, but these cells fail to follow the full mitotic sequence observed in the tailed Molgula occulta, nor do they undergo convergent extension (Takada et al., 2002). Finally, Ciona mutant aimless carries a null mutation in the gene encoding the essential planar cell polarity (PCP) component Prickle (Jiang et al., 2005). In the Ciona embryo prickle expression is limited to the notochord, and homozygous aimless embryos have defects in the intercalation of notochord cells, although markers of notochord differentiation are expressed normally. Despite these defects in notochord morphogenesis, aimless embryos gastrulate with no obvious defects, although they have greatly shortened anterior/posterior axes. Thus the PCP pathway is not required for the basic steps of involution and blastopore closure in Ciona. Whether this differs significantly from the role of the PCP pathway in vertebrate gastrulation is unclear. Xenopus embryos in which Prickle was knocked down with morpholinos have 
been described as having severe defects in mesoderm involution (Takeuchi et al., 2003).

However, PCP loss of function results in zebrafish appear to be more similar to Ciona, with a loss of mediolateral intercalation in axial mesoderm and a resulting shortened axes, but complete involution (Jessen et al., 2002; Veeman et al., 2003).

After the notochord has intercalated into a single file column of 40 cells, it undergoes considerable further morphogenesis, including cell shape changes and concomitant protein localization (and relocalization) to generate constricted apical domains at the interfaces between the stacked cells (see reviews (Denker and Jiang, 2012; Smith, 2018). Each notochord cell develops separate anterior and posterior apical domains (Denker et al., 2013). Extracellular lumens then form between the cells, centered at the apical domains. As these lumens expand they fuse to form a continuous inflated, hollow lumen running the length of the notochord and surrounded by the now epithelium-like notochord cells. Moreover, unlike many vertebrates, there is no fate distinction between separate notochord sheath and core cell types. At the same time that the apical domains are forming there is a repolarization of the PCP machinery. Early in notochord development PCP activity drives mediolateral intercalation (Jiang et al., 2005). However, at the completion of intercalation the polarity of PCP activity is repositioned to the anterior/posterior axis (Newman-Smith et al., 2015). The most obvious manifestation of the PCP-dependent anterior/posterior polarity of the notochord is the posterior localization of the nucleus, and the anterior localization of the PCP components Prickle and Strabismus, as well as myosin II (reviewed in (Smith, 2018)).

\section{Neural Induction and Neurulation}

In vertebrates, induction of the central nervous system initiates late in the blastula stage and continues through gastrulation. The vertebrate neural-inducing molecules are products of the dorsal lip of the blastopore, a tissue which is variously termed the Organizer in amphibians, the shield in teleosts, Hensen's node in chicks and the primitive streak in mammals. These inducers act on neighboring ectoderm to initiate the formation of the neural plate. A number of secreted factors have neural inducing activity, including FGF, and BMP inhibitors such as Noggin and Chordin [see review (Stern, 2005)]. The evolutionary similarities with the vertebrates are most evident in the morphology and morphogenesis of the ascidian larval nervous system (see review (Hudson, 2016)). While there are some parallels between ascidian and vertebrate neural induction, significant departures are also evident.

The Ciona neural plate has a remarkably simple structure with a grid-like arrangement of six rows and eight columns of cells that have distinct row and column identities (Hudson et al., 2007). The cells forming the plate come from 3 of the 4 quadrants of the 8-cell embryo and are induced in quite different ways. These include the a-lineage which gives rise to the anterior neural plate and goes on to make the anterior brain, palps and presumptive mouth (Veeman et al., 2010), the $A$-lineage which gives rise to posterior brain and the posterior nerve cord, and the $b$-lineage which gives rise to the dorsal-most row of cells in the posterior brain and posterior nerve chord (neural lineages reviewed in (Hudson, 2016)). Neural induction in Ciona starts at the 32-cell stage with the specification of the a-lineage (Bertrand et al., 2003) via vegetally expressed FGF9/16/20 (i.e., the ortholog of vertebrate FGFs 9, 16 and 20). During a-lineage neural specification, not only does contact between the inducer 
cells and the induced ectodermal cells appear to be important, but the area of contact appears to be essential in distinguishing between induced cells and neighboring non-induced cells (Tassy et al., 2006). Neural induction in the $b$-lineage is not well characterized, and the induction of $A$-lineage neural cells is very different than the a-lineage. The $A$-lineage of the neural plate becomes fate restricted at the 64-cell stage as the result of asymmetric cell divisions in which suppression of FGF signaling is required to suppress notochord fate (Yasuo and Hudson, 2007). While the Ciona embryo does express some secreted BMP inhibitors, none have been found to share the critical role in early neural induction seen in vertebrate embryos.

Ciona neurulation is stereotypically chordate, with a neural plate that forms a distinctive midline groove and rolls up to form a hollow, dorsal neural tube (Conklin, 1905a; Nicol and Meinertzhagen, 1988b; Satoh, 1978). The onset of neurulation is continuous with the end of gastrulation, with the neural folds first appearing at the posterior of the embryo on either side of the closing blastopore (Nicol and Meinertzhagen, 1988b). It is unclear whether this is simply a consequence of rapid early development or whether blastopore closure might be mechanistically important for early neurulation. Neural tube closure progresses from posterior to anterior (Hiroki Nishida, 1986). A transient chordoneural hinge can be seen in phalloidin-stained confocal stacks connecting the forming neural tube lumen to the remnant of the archenteron via the nearly-closed blastopore.

Neurulation first involves the furrowing and formation of a hinge point in the midline of the neural plate around which neural cells will bend as the midline cells constrict apically (Nicol and Meinertzhagen, 1988b). Convergence and extension movements narrow the mediolateral axis and lengthen the anterior-posterior axis of the neural plate as it rolls up (Nicol and Meinertzhagen, 1988b). Previously lateral and now dorsal cells meet at the midline and 'zipper' together to form a closed neural tube. The posterior to anterior zippering involves contraction of the apical domain of bilateral pairs of neural cells. These contractions are sequential and are punctuated by apical junction exchange and release from one pair of midline cells to another in a posterior to anterior direction (Hashimoto et al., 2015).

\section{Conclusion}

The presence of a number of traits in the tunicates, such as simple and stereotyped lineages, the extreme transparency of some ascidian species such as Phallusia, making them ideal for live imaging (Robin et al., 2011), their evolutionary affinity to vertebrates, and the development of a suite of genomic and imaging methods (Stolfi and Christiaen, 2012; Veeman and Reeves, 2015), has allowed them to make ongoing contributions to our understanding of gastrulation. Many questions remain, some specific to tunicate gastrulation, and others to chordate gastrulation in general. The apparent differences in the relative importance of endoderm involution in ascidian gastrulation in comparison to vertebrate models in which mesoderm and ectoderm are thought to play more prominent roles, raises the question of whether this is a derived feature of the ascidians. Has a complex gastrulation involving coordinated morphogenetic processes in different germ layers been lost in the ascidians, perhaps as a consequence of embryo simplification, or was a simple gastrulation driven predominantly by endoderm invagination a feature of the last common 
ancestor of the chordates? We have emphasized in this review the diversity of tunicate morphologies and apparent developmental strategies, although our detailed knowledge is limited to just a few species. Renewed investigation of lesser-studied tunicate clades, such as the salps, with modern methods may reveal new insights into the diversity of chordate developmental strategies, including those for gastrulation.

\section{Acknowledgments.}

We thank Hiroki Nishida for his helpful discussion of Oikopleura gastrulation. This work support by NIH grant HD038701 to WCS, and NIH grant HD085909 and NSF grant IOS 1456555 to MV.

\section{References}

Beane WS, Gross JM, McClay DR, 2006 RhoA regulates initiation of invagination, but not convergent extension, during sea urchin gastrulation. Dev Biol 292, 213-225. 10.1016/j.ydbio.2005.12.031 [PubMed: 16458878]

Bertrand V, Hudson C, Caillol D, Popovici C, Lemaire P, 2003 Neural tissue in ascidian embryos is induced by FGF9/16/20, acting via a combination of maternal GATA and Ets transcription factors. Cell 115, 615-27. [PubMed: 14651852]

Bourlat SJ, Rota-Stabelli O, Lanfear R, Telford MJ, 2009 The mitochondrial genome structure of Xenoturbella bocki (phylum Xenoturbellida) is ancestral within the deuterostomes. BMC Evol. Biol 9, 107 10.1186/1471-2148-9-107 [PubMed: 19450249]

Brown FD, Swalla BJ, 2012 Evolution and development of budding by stem cells: ascidian coloniality as a case study. Dev Biol 369, 151-162. 10.1016/j.ydbio.2012.05.038 [PubMed: 22722095]

Carlson M, Reeves W, Veeman M, 2015 Stochasticity and stereotypy in the Ciona notochord. Dev. Biol 397, 248-256. 10.1016/j.ydbio.2014.11.016 [PubMed: 25459659]

Chabry L, 1887 Contribution a l'embryologie normale et teratologique des Ascidie simples. J. Anat. Physiol

Chiba S, Jiang D, Satoh N, Smith WC, 2009 Brachyury null mutant-induced defects in juvenile ascidian endodermal organs. Development 136, 35-39. 10.1242/dev.030981 [PubMed: 19019990]

Chiba S, Miki Y, Ashida K, Wada MR, Tanaka KJ, Shibata Y, Nakamori R, Nishikata T, 1999 Interactions between cytoskeletal components during myoplasm rearrangement in ascidian eggs. Dev. Growth Differ 41, 265-272. [PubMed: 10400388]

Conklin, 1905a The organization and cell-lineage of the ascidian egg.

Conklin, 1905b Organ-forming stubstances in the eggs of ascidians. Biol. Bull VIII, 205-230.

Conklin, 1905c Mosaic development in ascidian eggs. J. Exp. Zool 2, 145-223.

Darwin C, 1871 The descent of man, and Selection in relation to sex, Vol 1 10.1037/12293-000

Delsuc F, Brinkmann H, Chourrout D, Philippe H, 2006 Tunicates and not cephalochordates are the closest living relatives of vertebrates. Nature 439, 965-968. 10.1038/nature04336 [PubMed: 16495997]

Delsuc F, Philippe H, Tsagkogeorga G, Simion P, Tilak MK, Turon X, López-Legentil S, Piette J, Lemaire P, Douzery EJP, 2018 A phylogenomic framework and timescale for comparative studies of tunicates. BMC Biol. 10.1186/s12915-018-0499-2

Dumollard R, Hebras C, Besnardeau L, McDougall A, 2013 Beta-catenin patterns the cell cycle during maternal-to-zygotic transition in urochordate embryos. Dev. Biol 384, 331-342. 10.1016/ j.ydbio.2013.10.007 [PubMed: 24140189]

Dumollard R, Minc N, Salez G, Aicha S. Ben, Bekkouche F, Hebras C, Besnardeau L, McDougall A, 2017 The invariant cleavage pattern displayed by ascidian embryos depends on spindle positioning along the cell's longest axis in the apical plane and relies on asynchronous cell divisions. Elife 6, 1-23. 10.7554/eLife. 19290

Ettensohn CA, 1999 Cell movements in the sea urchin embryo. Curr Opin Genet Dev 9, 461-465. 10.1016/S0959-437X(99)80070-7 [PubMed: 10449348] 
Ettensohn CA, 1985 Gastrulation in the sea urchin embryo is accompanied by the rearrangement of invaginating epithelial cells. Dev. Biol 112, 383-90. [PubMed: 4076547]

Fischer JL, 1992 The embryological œuvre of Laurent Chabry. Roux's Arch. Dev. Biol 10.1007/ BF00188709

Fujii S, Nishio T, Nishida H, 2008 Cleavage pattern, gastrulation, and neurulation in the appendicularian, Oikopleura dioica. Dev Genes Evol 218, 69-79. 10.1007/s00427-008-0205-4 [PubMed: 18236068]

Haeckel E, 1874 Anthropogenie oder Entwicklungsgeschichte des Menschen. Engelmann, Leipzig.

Hashimoto H, Robin FB, Sherrard KM, Munro EM, 2015 Sequential Contraction and Exchange of Apical Junctions Drives Zippering and Neural Tube Closure in a Simple Chordate. Dev. Cell 32, 241-255. 10.1016/j.devcel.2014.12.017 [PubMed: 25625209]

Hibino T, Nishikata T, Nishida H, 1998 Centrosome-attracting body: a novel structure closely related to unequal cleavages in the ascidian embryo. Dev. Growth Differ 40, 85-95. 10.1046/j.1440 [PubMed: 9563914]

Holland LZ, 2016 Tunicates. Curr Biol 26, R146-52. 10.1016/j.cub.2015.12.024 [PubMed: 26906481]

Hotta K, Mitsuhara K, Takahashi H, Inaba K, Oka K, Gojobori T, Ikeo K, 2007 A web-based interactive developmental table for the ascidian Ciona intestinalis, including 3D real-image embryo reconstructions: I. From fertilized egg to hatching larva. Dev. Dyn 236, 1790-1805. 10.1002/dvdy.21188 [PubMed: 17557317]

Hudson C, 2016 The central nervous system of ascidian larvae. Wiley Interdiscip. Rev. Dev. Biol 5, 538-561. 10.1002/wdev.239 [PubMed: 27328318]

Hudson C, Kawai N, Negishi T, Yasuo H, $2013 \beta$-Catenin-driven binary fate specification segregates germ layers in ascidian embryos. Curr. Biol 23, 491-495. 10.1016/j.cub.2013.02.005 [PubMed: 23453950]

Hudson C, Lotito S, Yasuo H, 2007 Sequential and combinatorial inputs from Nodal, Delta2/Notch and FGF/MEK/ERK signalling pathways establish a grid-like organisation of distinct cell identities in the ascidian neural plate. Development 134, 3527-3537. 10.1242/dev.002352 [PubMed: 17728350]

Hudson C, Sirour C, Yasuo H, 2016 Co-expression of Foxa.a, Foxd and Fgf9/16/20 defines a transient mesendoderm regulatory state in ascidian embryos. Elife 5, 1-17. 10.7554/eLife. 14692

Hudson C, Yasuo H, 2006 A signalling relay involving Nodal and Delta ligands acts during secondary notochord induction in Ciona embryos. Development 133, 2855-2864. 10.1242/dev.02466 [PubMed: 16835438]

Imai K, Takada N, Satoh N, Satou Y, 2000 (Beta)-Catenin Mediates the Specification of Endoderm Cells in Ascidian Embryos. Development 127, 3009-3020. [PubMed: 10862739]

Imai KS, 2003 Isolation and characterization of beta-catenin downstream genes in early embryos of the ascidian Ciona savignyi. Differentiation. 71, 346-360. 10.1046/j.1432-0436.2003.7106001.x [PubMed: 12919104]

Imai KS, Hudson C, Oda-Ishii I, Yasuo H, Satou Y, 2016 Antagonism between $\beta$-catenin and Gata.a sequentially segregates the germ layers of ascidian embryos. Development 143, 4167-4172. 10.1242/dev.141481 [PubMed: 27707797]

Iseto T, Nishida H, 1999 Ultrastructural studies on the centrosome-attracting body: electron-dense matrix and its role in unequal cleavages in ascidian embryos. Dev. Growth Differ 41, 601-609. 10.1046/j.1440-169x.1999.00457.x [PubMed: 10545032]

Jessen JR, Topczewski J, Bingham S, Sepich DS, Marlow F, Chandrasekhar A, Solnica-Krezel L, 2002 Zebrafish trilobite identifies new roles for Strabismus in gastrulation and neuronal movements. Nat. Cell Biol 4, 610-615. 10.1038/ncb828 [PubMed: 12105418]

Jiang D, Munro EM, Smith WC, 2005 Ascidian prickle regulates both mediolateral and anteriorposterior cell polarity of notochord cells. Curr Biol 15, 79-85. [PubMed: 15700379]

Keller R, Davidson LA, Shook DR, 2003 How we are shaped: the biomechanics of gastrulation. Differentiation 71, 171-205. 10.1046/j.1432-0436.2003.710301.x [PubMed: 12694202]

Kocot KM, Tassia MG, Halanych KM, Swalla BJ, 2018 Phylogenomics offers resolution of major tunicate relationships. Mol. Phylogenet. Evol 10.1016/j.ympev.2018.01.005 
Kowalevsky A, 1866 Entwicklungsgeschichte der einfachen Ascidien Mémoires l'Académie Impériale des Sci. St.-pétersbg VIIe Série.

Lemaire P, 2011 Evolutionary crossroads in developmental biology: the tunicates. Development 138, 2143-52. 10.1242/dev.048975 [PubMed: 21558365]

Lemaire P, Piette J, 2015 Tunicates: Exploring the sea shores and roaming the open ocean. A tribute to Thomas Huxley. Open Biol. 10.1098/rsob.150053

Logan CY, Miller JR, Ferkowicz MJ, Mcclay DR, 1999 Nuclear $\beta$-catenin is required to specify vegetal cell fates in the sea urchin embryo 357, 345-357.

Manni L, Anselmi C, Cima F, Gasparini F, Voskoboynik A, Martini M, Peronato A, Burighel P, Zaniolo G, Ballarin L, 2019 Sixty years of experimental studies on the blastogenesis of the colonial tunicate Botryllus schlosseri. Dev. Biol 448, 293-308. 10.1016/j.ydbio.2018.09.009 [PubMed: 30217596]

McDougall A, Sardet C, 1995 Function and characteristics of repetitive calcium waves associated with meiosis. Curr. Biol 5, 318-328. [PubMed: 7780742]

Miyawaki K, Yamamoto M, Saito K, Saito S, 2003 Nuclear localization of $\mathrm{N}_{\mathrm{L}}$-catenin in vegetal pole cells during early embryogenesis of the starfish Asterina pectinifera 121-128.

Munro EM, Odell G, 2002a Morphogenetic pattern formation during ascidian notochord formation is regulative and highly robust. Development 129, 1-12. [PubMed: 11782396]

Munro EM, Odell GM, 2002b Polarized basolateral cell motility underlies invagination and convergent extension of the ascidian notochord. Development 129, 13-24. [PubMed: 11782397]

Nicol D, Meinertzhagen IA, 1988a Development of the central nervous system of the larva of the ascidian, Ciona intestinalis L. I. The early lineages of the neural plate. Dev. Biol 130, 721-736. [PubMed: 3197929]

Nicol D, Meinertzhagen IA, 1988b Development of the central nervous system of the larva of the ascidian, Ciona intestinalis L. II. Neural plate morphogenesis and cell lineages during neurulation. Dev. Biol 130, 737-766. [PubMed: 3197930]

Nishida H, 2008 Development of the appendicularian Oikopleura dioica: culture, genome, and cell lineages. Dev. Growth Differ 50 Suppl 1, S239-56. 10.1111/j.1440-169X.2008.01035.x [PubMed: 18494706]

Nishida H, 1987 Cell lineage analysis in ascidian embryos by intracellular injection of a tracer enzyme. III. Up to the tissue restricted stage. Dev. Biol 121, 526-41. 10.1126/science.725606 [PubMed: 3582738]

Nishida H, 1986 Cell-Division Pattern during Gastrulation of the Ascidian, Halocynthia-Roretzi. Dev. Growth Differ 28, 191-201.

Nishida Hiroki, 1986 Cell Division Pattern during Gastrulation of the Ascidian, Halocynthia roretzi. Dev. Growth Differ 28, 191-201. 10.1111/j.1440-169X.1986.00191.x

Nishida H, Satoh N, 1985 Cell lineage analysis in ascidian embryos by intracellular injection of a tracer enzyme. II. The 16- and 32-cell stages. Dev Biol 110, 440-454. [PubMed: 4018407]

Nishida H, Satoh N, 1983 Cell lineage analysis in ascidian embryos by intracellular injection of a tracer enzyme. I. Up to the eight-cell stage. Dev Biol 99, 382-394. [PubMed: 6618008]

Nishida H, Sawada K, 2001 macho-1 encodes a localized mRNA in ascidian eggs that specifies muscle fate during embryogenesis. Nature 409, 724-729. 10.1038/35055568 [PubMed: 11217862]

Nishikata T, Hibino T, Nishida H, 1999 The centrosome-attracting body, microtubule system, and posterior egg cytoplasm are involved in positioning of cleavage planes in the ascidian embryo. Dev. Biol 209, 72-85. 10.1006/dbio.1999.9244 [PubMed: 10208744]

Nishikata T, Mita-Miyazawa I, Deno T, Satoh N, 1987 Muscle cell differentiation in ascidian embryos analysed with a tissue-specific monoclonal antibody. Development 99, 163-171. [PubMed: 2443339]

Okado H, Takahashi K, 1988 A simple "neural induction" model with two interacting cleavagearrested ascidian blastomeres. Proc. Natl. Acad. Sci. U. S. A 85, 6197-6201. 10.1073/ pnas.85.16.6197 [PubMed: 2457910]

Onai T, 2019 Canonical Wnt/ $\beta$-catenin and Notch signaling regulate animal/vegetal axial patterning in the cephalochordate amphioxus. Evol. Dev 21, 31-43. 10.1111/ede.12273 [PubMed: 30288919] 
Patalano S, Prulière G, Prodon F, Paix A, Dru P, Sardet C, Chenevert J, 2006 The aPKC-PAR-6-PAR-3 cell polarity complex localizes to the centrosome attracting body, a macroscopic cortical structure responsible for asymmetric divisions in the early ascidian embryo. J. Cell Sci 119, 1592-1603. 10.1242/jcs.02873 [PubMed: 16569661]

Picco V, Hudson C, Yasuo H, 2007 Ephrin-Eph signalling drives the asymmetric division of notochord/ neural precursors in Ciona embryos. Development 134, 1491-1497. 10.1242/dev.003939 [PubMed: 17344225]

Piette J, Lemaire P, 2015 Thaliaceans, the Neglected Pelagic Relatives of Ascidians: A Developmental and Evolutionary Enigma. Q Rev Biol 90, 117-145. [PubMed: 26285352]

Prodon F, Yamada L, Shirae-Kurabayashi M, Nakamura Y, Sasakura Y, 2007 Postplasmic/PEM RNAs: a class of localized maternal mRNAs with multiple roles in cell polarity and development in ascidian embryos. Dev. Dyn 236, 1698-1715. 10.1002/dvdy.21109 [PubMed: 17366574]

Racioppi C, Kamal AK, Razy-Krajka F, Gambardella G, Zanetti L, Di Bernardo D, Sanges R, Christiaen LA, Ristoratore F, 2014 Fibroblast growth factor signalling controls nervous system patterning and pigment cell formation in Ciona intestinalis. Nat. Commun 5, 1-17. 10.1038/ ncomms5830

Ricci L, Cabrera F, Lotito S, Tiozzo S, 2016 Redeployment of germ layers related TFs shows regionalized expression during two non-embryonic developments. Dev. Biol 416, 235-248. 10.1016/j.ydbio.2016.05.016 [PubMed: 27208394]

Robin FB, Dauga D, Tassy O, Sobral D, Daian F, Lemaire P, 2011 Time-Lapse Imaging of Live Phallusia Embryos for Creating 3D Digital Replicas. Cold Spring Harb. Protoc 2011, pdb.prot065847-pdb.prot065847. 10.1101/pdb.prot065847

Rothbacher U, Bertrand V, Lamy C, Lemaire P, 2007 A combinatorial code of maternal GATA, Ets and -catenin-TCF transcription factors specifies and patterns the early ascidian ectoderm. Development 134, 4023-4032. 10.1242/dev.010850 [PubMed: 17965050]

Sardet C, Speksnijder J, Jaffe L, 1989 Fertilization and ooplasmic movements in the ascidian egg. Development 249, 237-249.

Sardet C, Speksnijder J, Terasaki M, Chang P, Biologie U, De, Marine C, Cnrs URA, Paris U, Zoologique S, 1992 Polarity of the ascidian egg cortex before fertilization 237, 221-237.

Satoh N, 1978 Cellular morphology and architecture during early morphogenesis of the ascidian egg: an SEM study. Biol. Bull 155, 608-614. 10.2307/1540794 [PubMed: 749934]

Satoh N, Rokhsar D, Nishikawa T, 2014 Chordate evolution and the three-phylum system. Proc. R. Soc. B Biol. Sci 10.1098/rspb.2014.1729

Sherrard K, Robin F, Lemaire P, Munro E, 2010 Sequential activation of apical and basolateral contractility drives ascidian endoderm invagination. Curr Biol 20, 1499-1510. 10.1016/ j.cub.2010.06.075 [PubMed: 20691592]

Smith WC, 2018 Cellular Processes of Notochord Formation. Springer, Singapore, pp. 165-177. 10.1007/978-981-10-7545-2_15

Speksnijder JE, Sardet C, Jaffe LF, 1990 The activation wave of calcium in the ascidian egg and its role in ooplasmic segregation. J. Cell Biol 110, 1589-1598. 10.1083/jcb.110.5.1589 [PubMed: 2335565]

Stern CD, 2005 Neural induction: old problem, new findings, yet more questions. Development 132, 2007-2021. 10.1242/dev.01794 [PubMed: 15829523]

Stolfi A, Christiaen L, 2012 Genetic and genomic toolbox of the chordate Ciona intestinalis. Genetics 192, 55-66. 10.1534/genetics.112.140590 [PubMed: 22964837]

Stolfi A, Levine M, 2011 Neuronal subtype specification in the spinal cord of a protovertebrate. Development 138, 995-1004. 10.1242/dev.061507 [PubMed: 21303852]

Stolfi A, Ryan K, Meinertzhagen IA, Christiaen L, 2015 Migratory neuronal progenitors arise from the neural plate borders in tunicates. Nature 527, 371-374. 10.1038/nature15758 [PubMed: 26524532]

Stolfi A, Wagner E, Taliaferro JM, Chou S, Levine M, 2011 Neural tube patterning by Ephrin, FGF and Notch signaling relays. Development 138, 5429-5439. 10.1242/dev.072108 [PubMed: 22110057]

Sutton MF, 1960 The sexual development of Salpa fusiformis (Cuvier): Part I. J. Embryol. exp. Morph 8, 268-290. 
Swalla BJ, Jeffery WR, 1990 Interspecific hybridization between an anural and urodele ascidian: differential expression of urodele features suggests multiple mechanisms control anural development. Dev Biol 142, 319-334. [PubMed: 2124192]

Swalla BJ, Makabe KW, Satoh N, Jeffery WR, 1993 Novel genes expressed differentially in ascidians with alternate modes of development. Development 119, 307-318. [PubMed: 8287790]

Takada N, York J, Davis JM, Schumpert B, Yasuo H, Satoh N, Swalla BJ, 2002 Brachyury expression in tailless Molgulid ascidian embryos. Evol Dev 4, 205-211. [PubMed: 12054293]

Takeuchi M, Nakabayashi J, Sakaguchi T, Yamamoto TS, Takahashi H, Takeda H, Ueno N, 2003 The prickle-related gene in vertebrates is essential for gastrulation cell movements. Curr. Biol 13, 6749. [PubMed: 12699625]

Tassy O, Daian F, Hudson C, Bertrand V, Lemaire P, 2006 A quantitative approach to the study of cell shapes and interactions during early chordate embryogenesis. Curr. Biol 16, 345-358. 10.1016/ j.cub.2005.12.044 [PubMed: 16488868]

Tiozzo S, Brown FW, De Tomaso AW, 2008 No Title, in: Bosch T (Ed.), Stem Cells: From Hydra to Man. Springer, pp. 95-112.

Tokuoka M, Imai KS, Satou Y, Satoh N, 2004 Three distinct lineages of mesenchymal cells in Ciona intestinalis embryos demonstrated by specific gene expression. Dev. Biol 274, 211-224. 10.1016/ j.ydbio.2004.07.007 [PubMed: 15355799]

van Eekelen M, Runtuwene V, Overvoorde J, den Hertog J, 2010 RPTPalpha and PTPepsilon signaling via Fyn/Yes and RhoA is essential for zebrafish convergence and extension cell movements during gastrulation. Dev Biol 340, 626-639. 10.1016/j.ydbio.2010.02.026 [PubMed: 20188722]

Veeman M, Reeves W, 2015 Quantitative and in toto imaging in ascidians: working toward an imagecentric systems biology of chordate morphogenesis. Genesis 53, 143-159. 10.1002/dvg.22828 [PubMed: 25262824]

Veeman MT, Newman-Smith E, El-Nachef D, Smith WC, 2010 The ascidian mouth opening is derived from the anterior neuropore: Reassessing the mouth/neural tube relationship in chordate evolution. Dev. Biol 344, 138-149. 10.1016/j.ydbio.2010.04.028 [PubMed: 20438724]

Veeman MT, Slusarski DC, Kaykas A, Louie SH, Moon RT, 2003 Zebrafish prickle, a modulator of noncanonical Wnt/Fz signaling, regulates gastrulation movements. Curr. Biol 13, 680-5. [PubMed: 12699626]

Wang W, Niu X, Stuart T, Jullian E, Mauck WM, Kelly RG, Satija R, Christiaen L, 2019 A single-cell transcriptional roadmap for cardiopharyngeal fate diversification. Nat. Cell Biol 21, 674-686. 10.1038/s41556-019-0336-z [PubMed: 31160712]

Whittaker JR, 1973 Segregation during ascidian embryogenesis of egg cytoplasmic information for tissue-specific enzyme development. Proc. Natl. Acad. Sci. U. S. A 70, 2096-2100. 10.1073/ pnas.70.7.2096 [PubMed: 4198663]

Wieschaus E, 2000 A Genetic Link between Morphogenesis and Cell Division during Formation of the Ventral Furrow in Drosophila 101, 523-531.

Wikramanayake AH, Hong M, Lee PN, Pang K, Byrum CA, Bince JM, Xu R, Martindale MQ, 2003 An ancient role for nuclear beta-catenin in the evolution of axial polarity and germ layer segregation. Nature 426, 446-450. 10.1038/nature02113 [PubMed: 14647383]

WoRMS - World Register of Marine Species [WWW Document], 2019 . Available from http:// www.marinespecies.org. URL http://www.marinespecies.org

Yasuo H, Hudson C, 2007 FGF8/17/18 functions together with FGF9/16/20 during formation of the notochord in Ciona embryos. Dev. Biol 302, 92-103. 10.1016/j.ydbio.2006.08.075 [PubMed: 17022960]

Brusca RC, and Brusca GJ (1990). Invertebrates (Sunderland, MA: Sinauer).

Denker E, Bocina I, and Jiang D (2013). Tubulogenesis in a simple cell cord requires the formation of bi-apical cells through two discrete Par domains. Development 140, 2985-2996. [PubMed: 23760958]

Denker E, and Jiang D (2012). Ciona intestinalis notochord as a new model to investigate the cellular and molecular mechanisms of tubulogenesis. Semin Cell Dev Biol 23, 308-319. [PubMed: 22465520] 
Fiuza U-M, Negishi T, Rouan A, Yasuo H, and Lemaire P (2018). Nodal and Eph signalling relay drives the transition between apical constriction and apico-basal shortening during ascidian endoderm invagination. bioRxiv.

Newman-Smith E, Kourakis MJ, Reeves W, Veeman M, and Smith WC (2015). Reciprocal and dynamic polarization of planar cell polarity core components and myosin. Elife 4, e05361. [PubMed: 25866928]

Smith WC (2018). Cellular Processes of Notochord Formation. Adv Exp Med Biol 1029, 165-177. [PubMed: 29542089] 


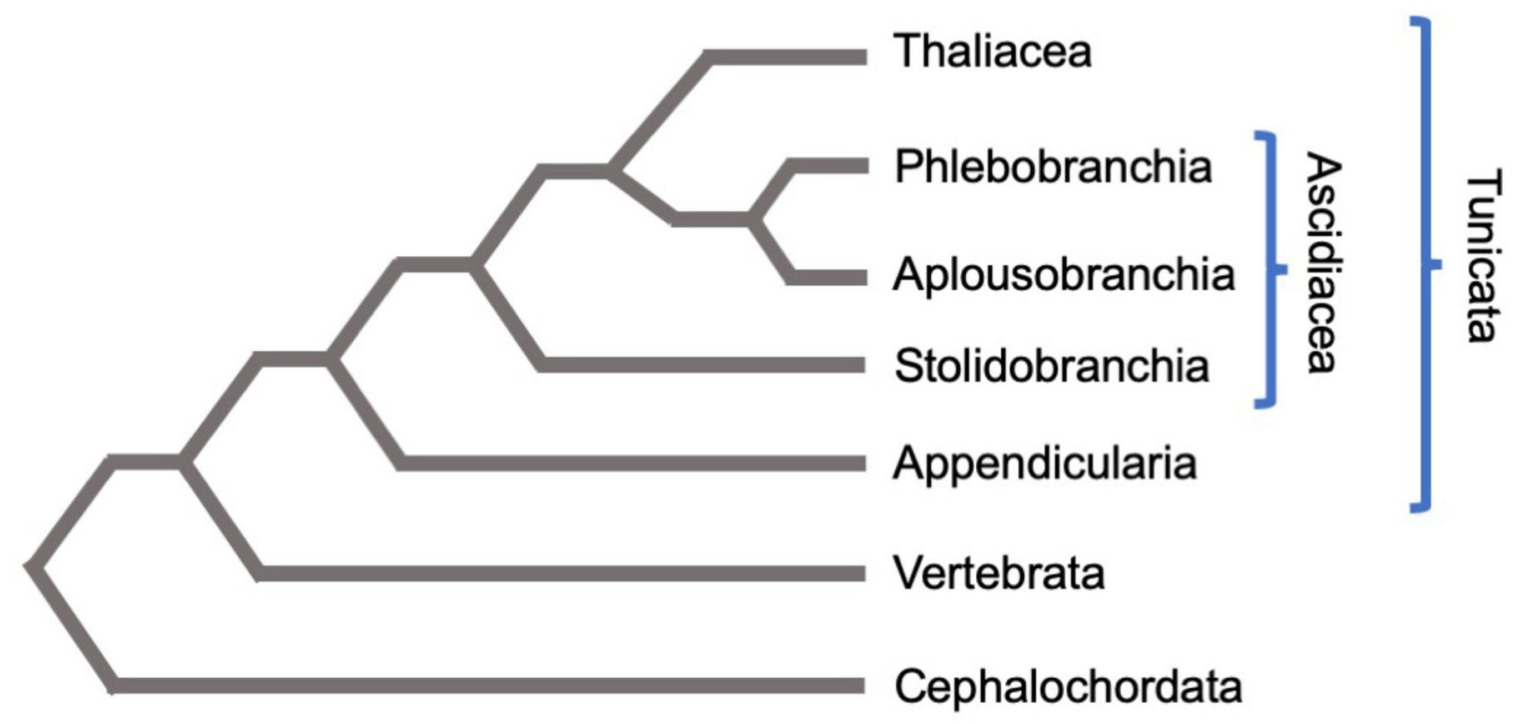

Figure 1.

Phylogenetic relationships between the chordates, with emphasis on the tunicate clade (based on Kocot et al. 2018 and Delsuc et al. 2018). Tunicates and vertebrates share a more recent common ancestor than either do with cephalochordates. Because larvaceans (Appendicularia) are basal in the Tunicata, the ancestral tunicate may have been pelagic. The Ascidiacea are revealed to be paraphyletic due to the placement of the Thaliacea as sister to Phlebobranchia/Aplousiobranchia. Branch lengths are not to scale. 

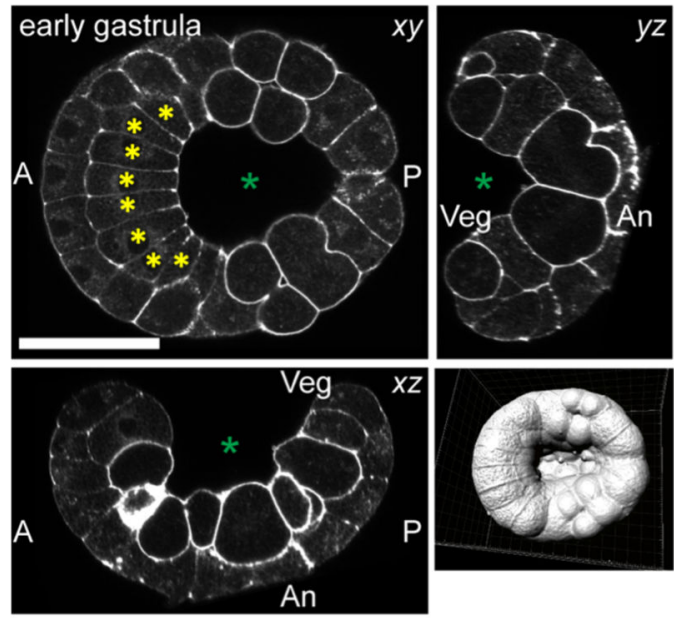
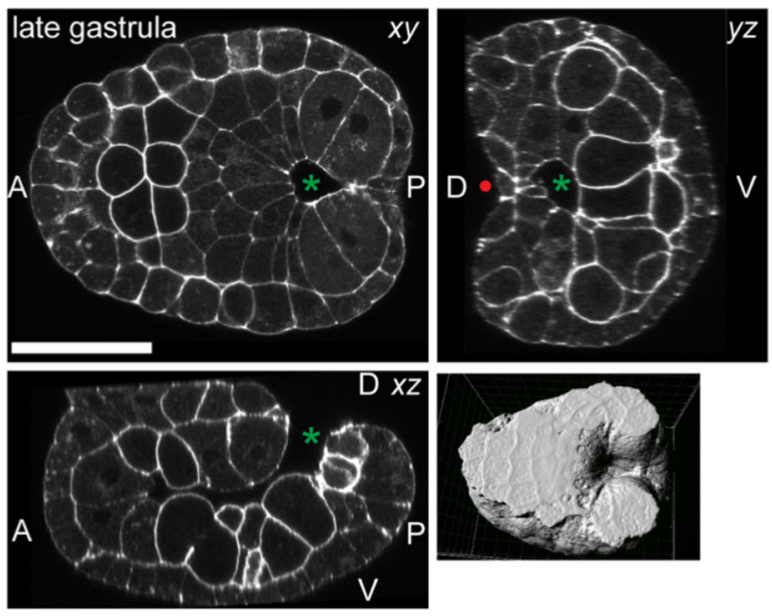

Figure 2. Ciona gastrulation.

Confocal images of gastrulating Ciona robusta embryos at the stages indicated. The panels on the left are derived from confocal stacks of a phalloidin-stained embryo early in gastrulation, with orthogonal $x y, x z$ and $y z$ sections shown. The surface-rendered view of the stack is from the vegetal side. The green asterisk marks the open blastopore/archenteron. Yellow asterisks mark notochord precursor cells. The panels on the right show an older embryo near the end of gastrulation/beginning of neurulation. The red dot indicates the neural groove just starting to form on the posterior dorsal side. Scale bars $=50 \mu \mathrm{m}$. 


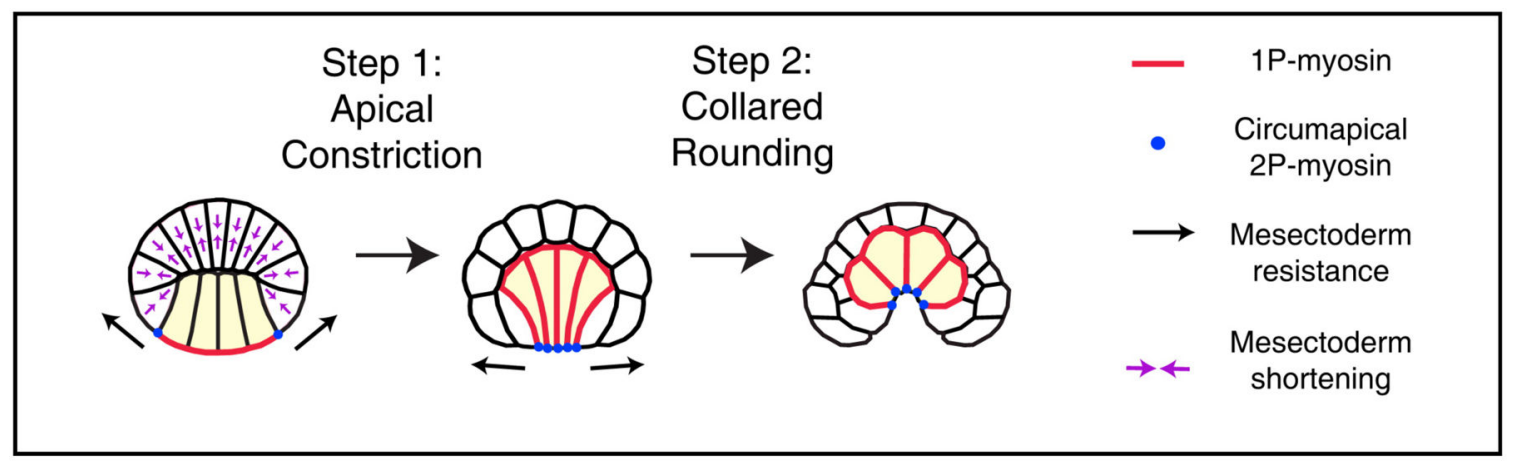

Figure 3.

Two-step model for endoderm invagination in ascidian development. Figure from (Sherrard et al., 2010). 


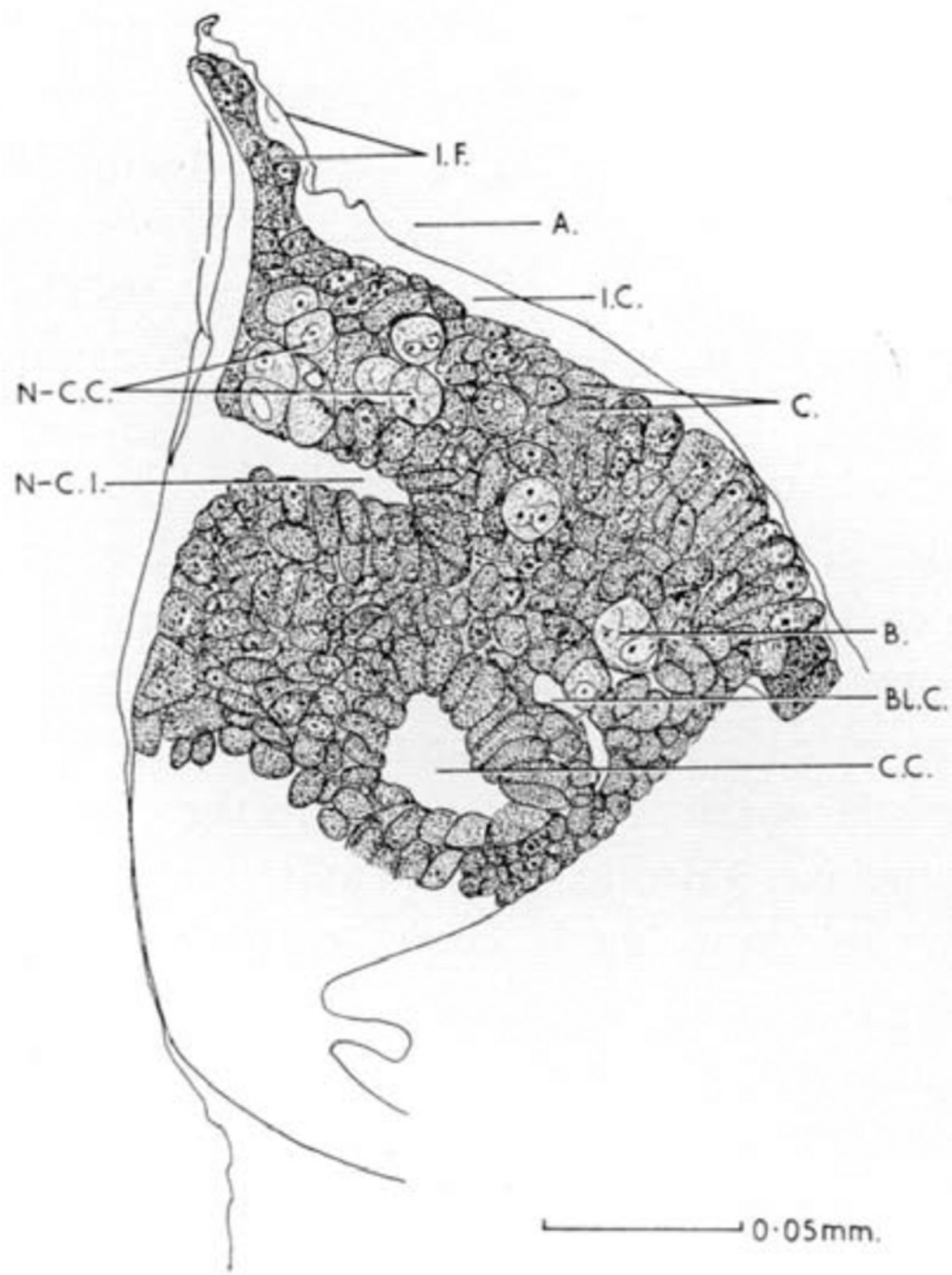

Figure 4.

Blastophore of Salpa fusiformis. Image from (Sutton, 1960). The precise developmental age of the blastophore is not known. Abbreviations: I.F., Incubation fold; A., atrium; I.C., incubation cavity; C., calymnocyte; B., blastomere; C.C., central cavity; N-C.I., neurochordal invagination; N-C.C. Neuro-chordal cells. 